\title{
Using Gravitational Lensing to study HI clouds at high redshift
}

\author{
Tarun Deep Saini, \\ Inter-University Center for Astronomy 8 Astrophysics, Puné 411 007, India; saini@iucaa.ernet.in \\ Somnath Bharadwaj \\ Department of Physics and Meteorology \& Center for Theoretical Studies, I.I.T. Kharagpur, 721 \\ 302,India; somnath@phy.iitkgp.ernet.in \\ and \\ Shiv K. Sethi \\ Harish Chandra Institute, Chhatnag Road, Jhusi, Allahabad 211 019, India; sethi@mri.ernet.in
}

\begin{abstract}
We investigate the possibility of detecting HI emission from gravitationally lensed HI clouds (akin to damped Lyman- $\alpha$ clouds) at high redshift by carrying out deep radio observations in the fields of known cluster lenses. Such observations will be possible with present radio telescopes only if the lens substantially magnifies the flux of the HI emission. While at present this holds the only possibility of detecting the HI emission from such clouds, it has the disadvantage of being restricted to clouds that lie very close to the caustics of the lens. We find that observations at a detection threshold of $50 \mu \mathrm{Jy}$ at $320 \mathrm{MHz}$ (possible with the GMRT) have a greater than $20 \%$ probability of detecting an $\mathrm{HI}$ cloud in the field of a cluster, provided the clouds have HI masses in the range $5 \times 10^{8} \mathrm{M}_{\odot} \leq M_{\mathrm{HI}} \leq 2.5 \times 10^{10} \mathrm{M}_{\odot}$. The probability of detecting a cloud increases if they have larger HI masses, except in the cases where the number of HI clouds in the cluster field becomes very small. The probability of a detection at $610 \mathrm{MHz}$ and $233 \mathrm{MHz}$ is comparable to that at $320 \mathrm{MHz}$, though a definitive statement is difficult owing to uncertainties in the HI content at the redshifts corresponding to these frequencies. Observations at a detection threshold of $2 \mu \mathrm{Jy}$ (possible in the future with the SKA) are expected to detect a few HI clouds in the field of every cluster provided the clouds have $\mathrm{HI}$ masses in the range $2 \times 10^{7} \mathrm{M}_{\odot} \leq M_{\mathrm{HI}} \leq 10^{9} \mathrm{M}_{\odot}$. Even if such observations do not result in the detection of HI clouds, they will be able to put useful constraints on the HI content of the clouds.
\end{abstract}

subject headings: cosmology: gravitational lensing; theory: galaxies; large-scale structure of the universe; radio lines: HI 


\section{Introduction}

Quasar spectra reveal a variety of absorption features superposed on the continuum emission, indicating the existence of HI clouds with a wide range of neutral hydrogen column densities distributed at different redshift. Among these the damped Lyman- $\alpha$ clouds (DLA) which have HI column densities in the range $2 \times 10^{20} \leq N_{\mathrm{HI}}\left(\mathrm{cm}^{-2}\right) \leq 10^{22}$ are the main repository of neutral hydrogen at high redshift $(z \simeq 3)$. It has been speculated that these systems are the progenitor of present day spiral galaxies (for details see Wolfe 1995 and references therein).

In addition to the column densities, absorption studies have determined the detailed velocity structure of the DLAs (Prochaska \& Wolfe 1998), and different models have been proposed for DLAs in order to explain these observations. The DLAs have been modeled as thick rotating disks with rotation velocities in the range $200-300 \mathrm{~km} \mathrm{~s}^{-1}$ (Prochaska \& Wolfe 1998). Another model proposes that DLAs are made up of gaseous protogalactic clumps undergoing in-fall (Haehnelt, Steinmetz \& Rauch 1997).

Despite very detailed absorption studies, the exact nature of the DLAs is still not fully understood. A part of the uncertainty arises from the fact that absorption studies give information along a single line of sight through a DLA, and this cannot be used to determine the total HI content or the physical extent of these systems. In a few cases there exist multiple lensed images of a quasar from which we can infer that the angular size of the DLAs is $\simeq 3^{\prime \prime}$ (Smette 1995 and reference therein). Observations of DLAs in HI absorption and deep-imaging of the DLA fields in the optical band have also been used to infer the size of DLAs (Lane et al. 2000; Fynbo et al. 2000; Fynbo et al. 1999; Moller et al. 1998; Warren \& Moller 1996; Moller \& Warren 1993; Briggs et al. 1989; Briggs 1988), but such observations only give lower limits on the physical size of DLAs.

In a few cases it has been possible to use deep optical imaging and spectroscopy to identify counterparts (in emission) of the systems that produce the damped Lyman- $\alpha$ lines. While most of these observations have been performed on low redshift DLA fields (see e.g. Brun et al. 1997 and references therein) there also exist several cases of positive detection of DLAs at high redshift (Kulkarni et al. 2000; Djorgovski et al. 1996; Fynbo et al. 2000; Fynbo et al. 1999; Moller et al. 1998; Warren \& Moller 1996; Moller \& Warren 1993).

Here we consider the possibility of detecting HI emission from hydrogen clouds whose column densities are sufficiently high to qualify as DLAs, if they were located along the line of sight to quasars. This allows the properties of the HI clouds to be inferred from the observed properties of DLAs. These observations will also enable the total HI content of the DLAs to be determined. Unfortunately, the very small angular extent of the DLAs leads us to expect HI flux from these clouds to be below the detection threshold of existing radio telescopes.

Optical observations of background galaxies (typically $\lesssim 1^{\prime \prime}$ ) lensed by cluster of galaxies reveal arc-like images which could be as large as $20^{\prime \prime}$ (see e.g. Schneider et al. 1992). Detailed models have been constructed for these cluster lenses; and it is possible to predict the magnification 
of the image of HI clouds if they were located at different positions behind the cluster. The lensing of HI clouds by clusters of galaxies holds the possibility of magnifying them sufficiently, rendering the HI emission detectable by presently available radio telescopes. In this paper we investigate the possibility of detecting the redshifted $1420 \mathrm{MHz} \mathrm{HI}$ emission from lensed HI clouds by carrying out deep radio observations centered on known cluster lenses.

Our investigation is largely motivated by the fact that the Giant Meterwave Radio Telescope (GMRT; G. Swarup et al. 1991) which has several bands in the frequency range 150-1420 MHz has recently started functioning. In this paper we consider frequency bands of width $16 \mathrm{MHz}$ centered at 233, 320 and $620 \mathrm{MHz}$. These correspond to the redshifted $1420 \mathrm{MHz}$ emission from $\mathrm{HI}$ at $z \simeq 1.3,3.4$ and 5.1 respectively. Most of the discussion in this paper refers to $320 \mathrm{MHz}$ which we have used as the fiducial frequency. In addition we use a spatially flat $\Omega_{m}=1$ model, and a Hubble parameter $H_{0}=100 h \mathrm{~km} \mathrm{~s}^{-1}$. We shall use $h=0.5$ whenever a numerical result is reported.

The absorption studies of DLAs have yielded a maximum HI column density of $\lesssim 10^{22}$ (Lanzetta, Wolfe \& Turnshek 1995). It is possible that clouds with higher column densities contain larger amount of dust that obscures the background quasar, in which case these clouds would not be detected in absorption (Fall \& Pei 1995). This possibility has been taken into account while analyzing the possibility of detecting the HI emission by setting the maximum HI column density of the clouds to a value which is a few times larger than the maximum HI column densities observed in absorption.

In $\S 2$ we review the observed properties of HI clouds which are relevant for our analysis and describe two simple models for the HI cloud mass and flux distribution. In $\S 3$ we briefly discuss a few relevant features of gravitational lensing by a cluster of galaxies and outline the method of our calculation. We present our results in $\S 4$ and in $\S 5$ we give our conclusions.

\section{Mass and flux distribution of $\mathrm{HI}$ clouds}

We begin this section with a brief discussion of some of the observational features of DLAs which we use to determine the properties of the distribution of HI clouds.

Absorption studies directly give the velocity dispersion $\Delta V$ of the HI along lines of sight through DLAs and these observations indicate rotational velocities in the range $200-250 \mathrm{~km} \mathrm{~s}^{-1}$ (Prochaska \& Wolfe 1998). This along with the HI column density can be used to calculate the specific intensity of the redshifted $1420 \mathrm{MHz}$ emission from the $\mathrm{HI}$ along the line of sight through a HI cloud. This gives (for derivation see Appendix A):

$$
I_{\nu}=40 \mu \mathrm{Jy} \operatorname{arcsec}^{-2}(1+z)^{-3}\left(\frac{N_{\mathrm{HI}}}{10^{22} \mathrm{~cm}^{-2}}\right)\left(\frac{200 \mathrm{~km} \mathrm{~s}^{-1}}{\Delta V}\right) .
$$

Integrating the specific intensity over the angular extent of the HI cloud gives the total flux from 
the cloud:

$$
F_{\nu} \propto \int N_{\mathrm{HI}}(\boldsymbol{\beta}) d^{2} \beta
$$

where $\boldsymbol{\beta}$ (in arc-seconds) refers to different angular positions on the sky, and the integral is over the angular extent of the HI cloud.

In a situation where there is gravitational lensing a source which would otherwise appear at $\boldsymbol{\beta}$ is seen at a different angular position $\boldsymbol{\theta}$ (see e.g. Schneider et al. 1992), and the flux from the lensed image of the HI cloud can be calculated by performing a similar integration over the angular extent of the image as in Eq. (2).

Estimating the flux expected from a HI cloud (lensed or unlensed) requires knowledge about its angular extent and the variation in the column density across the HI cloud. As these facts are not available, we have adopted two simple models for our calculations in this paper. We describe these models below.

\subsection{Uniform disk (UD)}

In this model the HI clouds are assumed to be face on circular disks, all of the same physical radius $r_{\text {DLA }}$ and with uniform column density across the disk. We consider different values of $r_{\text {DLA }}$ in the range $10 \mathrm{kpc}$ to $30 \mathrm{kpc}$. The solid angle subtended by a $\mathrm{HI}$ cloud is $\Delta \Omega_{\mathrm{DLA}}=\pi r_{\mathrm{DLA}}^{2} / d_{s}^{2}(z)$ where $d_{s}(z)$ is the angular diameter distance to the HI cloud (see e.g. Peebles 1993). In this model the flux from an unlensed $\mathrm{HI}$ cloud is $F_{\nu}=I_{\nu} \Delta \Omega_{\mathrm{DLA}}$ and we have, for $\nu=320 \mathrm{MHz}$,

$$
F_{\nu}=12 \mu \mathrm{Jy}^{2}\left(\frac{N_{\mathrm{HI}}}{10^{22} \mathrm{~cm}^{-2}}\right)\left(\frac{200 \mathrm{~km} \mathrm{~s}^{-1}}{\Delta V}\right)\left(\frac{r_{\mathrm{DLA}}}{10 \mathrm{kpc}}\right)^{2} \text {. }
$$

The distribution of the column densities of the HI clouds is assumed to be a power law $F\left(N_{\mathrm{HI}}\right)=$ $B N_{\mathrm{HI}}^{-\alpha}$ with values in the range $2 \times 10^{20} \mathrm{~cm}^{-2}<N_{\mathrm{HI}}<10^{22} \mathrm{~cm}^{-2}$, where $F\left(N_{\mathrm{HI}}\right) d N_{\mathrm{HI}}$ gives the number of HI clouds per unit volume with column density in the range $d N_{\mathrm{HI}}$. Observations indicate that the column density distribution changes with redshift implying that both $\alpha$ and $B$ change with $z$. The DLA population shows a marked increase in the HI column densities at higher redshifts (Lanzetta et al. 1995) and 75\% of the DLAs in the redshift range $3 \leq z \leq 3.5$ have column densities

$N_{\mathrm{HI}} \geq 10^{21} \mathrm{~cm}^{-2}$. This is consistent with $\alpha=0.5$. In our work we consider values of $\alpha$ in the range 0.5 to 1.0 .

In this model the HI mass of a cloud is given by

$$
M_{\mathrm{HI}}=2.5 \times 10^{10} \mathrm{M}_{\odot}\left(\frac{r_{\mathrm{DLA}}}{10 \mathrm{kpc}}\right)^{2}\left(\frac{N_{\mathrm{HI}}}{10^{22} \mathrm{~cm}^{-2}}\right) .
$$

This relation permits us to interchangeably use either a column density distribution function or a HI mass distribution function $f\left(M_{\mathrm{HI}}\right)=A M_{\mathrm{HI}}^{-\alpha}$ where $f\left(M_{\mathrm{HI}}\right) d M_{\mathrm{HI}}$ is the number of HI clouds per 
unit volume with $\mathrm{HI}$ mass in the range $d M_{\mathrm{HI}}$. The mass range corresponding to the column density range $2 \times 10^{20} \mathrm{~cm}^{-2}<N_{\mathrm{HI}}<10^{22} \mathrm{~cm}^{-2}$ depends on the value of $r_{\mathrm{DLA}}$, and for $r_{\mathrm{DLA}}=10 \mathrm{kpc}$ we have $M_{\mathrm{HI}}[\min ]=5 \times 10^{8} \mathrm{M}_{\odot}$ and $M_{\mathrm{HI}}[\max ]=2.5 \times 10^{10} \mathrm{M}_{\odot}$. At any redshift $z$ the HI mass distribution function can be used to calculate the HI density

$$
\int_{M_{\mathrm{HI}}[\min ]}^{M_{\mathrm{HI}}[\max ]} M_{\mathrm{HI}} f\left(M_{\mathrm{HI}}\right) d M_{\mathrm{HI}}=\rho_{c} \Omega_{\mathrm{HI}},
$$

where $\rho_{c}$ is the critical density at redshift $z$ and $\Omega_{\mathrm{HI}}$ is the contribution to the density parameter from the HI in DLAs at that redshift. Absorption studies (Lanzetta et al. 1995) have shown that in a flat cosmological model, in the redshift range $z \lesssim 3.5$, the total mass density contributed by the HI in DLAs can be fitted by the function

$$
\Omega_{\mathrm{HI}}(z)=0.19 \times 10^{-3} h^{-1} \exp (0.83 z) .
$$

We use this to fix the normalization constant $A$ for the HI mass distribution function.

\subsection{Exponential disk (ED)}

Recent studies of the velocity structure of the DLAs show that the HI density across the cloud is consistent with a thick, rotating disk of exponential profile (Prochaska \& Wolfe 1998)

$$
n(R, Z)=n_{0} \exp \left(-\frac{R}{R_{\mathrm{d}}}-\frac{|Z|}{Z_{\mathrm{d}}}\right),
$$

where $n(R, Z)$ is the number density of $\mathrm{HI}$ atoms in the disk, $R$ is the radial coordinate in the plane of the disk and $Z$ is the coordinate along the thickness of the disk. Observations suggest that disks have $R_{\mathrm{d}} / Z_{\mathrm{d}} \simeq 3$, and are rotating with $v_{c} \simeq 200-250 \mathrm{~km} \mathrm{~s}^{-1}$. Excluding the effect of rotation, the gas in the disks has negligible velocity dispersion. Absorption studies do not fix $R_{\mathrm{d}}$ and they are only mildly dependent on the central column density $N_{\mathrm{HI}}(0)=2 Z_{\mathrm{d}} n_{0}$. In our analysis we assume that all the disks are observed face-on. As a consequence the column density distribution in each HI cloud is circularly symmetric around the center and falls off exponentially in the radial direction. For $\nu=320 \mathrm{MHz}$, the flux from a HI cloud is

$$
F_{\nu}=24 \mu \mathrm{Jy} h^{2}\left(\frac{N_{\mathrm{HI}}(0)}{10^{22} \mathrm{~cm}^{-2}}\right)\left(\frac{200 \mathrm{~km} \mathrm{~s}^{-1}}{\Delta V}\right)\left(\frac{R_{\mathrm{d}}}{10 \mathrm{kpc}}\right)^{2},
$$

and the HI mass is

$$
M_{\mathrm{HI}}=6 \times 10^{10} \mathrm{M}_{\odot}\left(\frac{N_{\mathrm{HI}}(0)}{10^{22} \mathrm{~cm}^{-2}}\right)\left(\frac{R_{\mathrm{d}}}{10 \mathrm{kpc}}\right)^{2} .
$$

We choose the central column density, $N_{\mathrm{HI}}(0)$ to have a fixed value in the range $5 \times 10^{21} \mathrm{~cm}^{-2}$ to $2 \times 10^{22} \mathrm{~cm}^{-2}$ and allow $R_{\mathrm{d}}$ to vary from $2 \mathrm{kpc}$ to $10 \mathrm{kpc}$. For a fixed value of $N_{\mathrm{HI}}(0)=10^{22} \mathrm{~cm}^{-2}$ this corresponds to a mass range $M_{\mathrm{HI}}[\min ]=2 \times 10^{9} \mathrm{M}_{\odot}$ and $M_{\mathrm{HI}}[\max ]=5 \times 10^{10} \mathrm{M}_{\odot}$. The treatment of the mass distribution function for the ED model has been carried out in exactly the same way as for the UD model, and we consider values in the range 0.5 to 1.0 for the slope $\alpha$. 


\subsection{The flux distribution of HI clouds}

For both the UD and the ED model the HI mass distribution function can be converted to a flux distribution function using either Eqs. (3) and (4) or Eqs. (8) and (9). This requires assuming a value for $\Delta V$ which we take to be $200 \mathrm{~km} \mathrm{~s}^{-1}$. The GMRT channel at $320 \mathrm{MHz}(z=3.4)$ has a bandwidth of $16 \mathrm{MHz}$. This corresponds to a redshift range of 3.33 to 3.55. We use the flux distribution function to calculate the number of clouds per unit flux per $\operatorname{arcsec}^{2}$ within the redshift range covered by the GMRT bandwidth.

For the UD model where the value of $r_{\text {DLA }}$ is fixed this gives us

$$
\begin{aligned}
& \frac{d^{2} n}{d F_{\nu} d \Omega} \simeq 1.2 \times 10^{-3} \mu \mathrm{Jy}^{-1} \operatorname{arcsec}^{-2} \times \\
& (2-\alpha)\left(\frac{r_{\mathrm{DLA}}}{10 \mathrm{kpc}}\right)^{-4+2 \alpha}\left(\frac{F_{\nu}}{1 \mu \mathrm{Jy}}\right)^{-\alpha} .
\end{aligned}
$$

For this model the different fluxes correspond to different values of the column density. For the ED model where the central column density $N_{\mathrm{HI}}(0)$ is fixed, the number of clouds per unit flux per $\operatorname{arcsec}^{2}$ is

$$
\begin{aligned}
& \frac{d^{2} n}{d F_{\nu} d \Omega} \simeq 4.25 \times 10^{-4} \mu \mathrm{Jy}^{-1} \operatorname{arcsec}^{-2} \times \\
& (2-\alpha)\left(\frac{N_{\mathrm{HI}}(0)}{10^{22} \mathrm{~cm}^{-2}}\right)^{-2+\alpha}\left(\frac{F}{1 \mu \mathrm{Jy}}\right)^{-\alpha} .
\end{aligned}
$$

(In Eqs. (10) and (11) the normalization has a weak dependence on the parameter $\alpha$, which is not shown for brevity) In this model the variation of flux corresponds to different values of the radial scale length $R_{\mathrm{d}}$. For $\nu=320 \mathrm{MHz}$, the total number of clouds expected above a given flux in a $1^{\prime} \times 1^{\prime}$ region of the sky and within the GMRT bandwidth is shown in Figure 1 for the UD and ED models.

\section{Gravitational Lensing by clusters}

Here we give a brief summary of the salient features of gravitational lensing relevant to this work. A more thorough exposition can be found in Schneider et al. (1992).

An object which is gravitationally lensed appears at a position which is different from its unlensed position. This information is encoded in the lens equation which relates the unperturbed position of the source to its perturbed position. If the angular coordinates of the source are $\boldsymbol{\beta}$ and the angular coordinates of the image are $\boldsymbol{\theta}$, then the lens equation is given by

$$
\boldsymbol{\beta}=\boldsymbol{\theta}-\nabla \psi(\boldsymbol{\theta})
$$


The dimensionless relativistic lens potential $\psi$ satisfies the two-dimensional Poisson equation $\nabla^{2} \psi(\boldsymbol{\theta})=$ $2 \kappa(\boldsymbol{\theta})$, where the convergence $\kappa(\boldsymbol{\theta})=\Sigma(\boldsymbol{\theta}) / \Sigma_{\mathrm{cr}}$ and $\Sigma(\boldsymbol{\theta})$ is the two-dimensional surface mass density of the lens, $\Sigma_{\mathrm{cr}}=\left(c^{2} / 4 \pi G\right)\left(d_{s} / d_{l} d_{l s}\right)$ being the critical density. The quantities $d_{s}$, $d_{l}$, and $d_{l s}$ are the angular-diameter distances from the observer to source, the observer to lens, and the lens to source respectively. Gravitational lensing does not effect the specific intensity along a light ray. The flux received by an observer is proportional to the solid angle subtended by the image at the observer; and since the solid angle of the image after lensing is, in general, different from that of the source, an observer can receive more (or less) flux in the lensed case than in the unlensed case.

The shape and size of the image are related to those of the source by the transformation matrix $M_{i j}^{-1}=\partial \beta_{i} / \partial \theta_{j}$. The infinitesimal area $d^{2} \theta$ in the image plane is mapped to an area $d^{2} \beta=\mu^{-1}(\boldsymbol{\theta}) d^{2} \theta$ in the source plane, where $\mu=\operatorname{det}\left[M_{i j}\right]$ is the magnification. The regions (curves) in the source plane where $\operatorname{det}\left[\partial \beta_{i} / \partial \theta_{j}\right]=0$ are called caustics. Magnification is infinite for point sources which are placed on the caustics. For finite sources the amplification remains finite, though the caustics still remain the points in the source plane where the magnification is large.

For this paper we model clusters of galaxies as single component Pseudo Isothermal Elliptical Mass Distribution (PIEMD) after Kassiola \& Kovner (1993). These are characterized by a core radius $R_{\mathrm{c}}$, one-dimensional velocity dispersion $\sigma$, and ellipticity parameter $\epsilon$. In addition to these parameters, the lens redshift $z_{l}$ and the source redshift $z_{s}$ completely specify the dimensionless potential $\psi$. Real clusters can have substantial sub-structure and are best modeled as multicomponent PIEMDs, but for simplicity we consider only single component lenses.

For our case the source redshift, $z_{s}=\nu_{e} / \nu_{o}-1$ with $\nu_{e}=1420 \mathrm{MHz}$, and $\nu_{o}=\{233,320,620\} \mathrm{MHz}$. For our study we consider the following range of parameters: $1200 \mathrm{~km} \mathrm{~s}^{-1} \leq \sigma \leq 1800 \mathrm{~km} \mathrm{~s}^{-1}$; $40 \mathrm{kpc} \leq R_{\mathrm{c}} \leq 60 \mathrm{kpc} ; 0 \leq \epsilon \leq 0.3$, and $0.1 \leq z_{l} \leq 0.3$. This choice of parameters is motivated by the observed properties of known clusters and their abundances (e.g. Mazure et al., 1996).

\subsection{Lensing of $\mathrm{HI}$ clouds}

The flux from a lensed HI cloud can be expressed in terms of the source coordinates $\boldsymbol{\beta}$ and the magnification $\mu(\boldsymbol{\beta})$ as:

$$
\begin{aligned}
F_{\nu}= & 40 \mu \mathrm{Jy} \operatorname{arcsec}^{-2}(1+z)^{-3}\left(\frac{200 \mathrm{~km} \mathrm{~s}^{-1}}{\Delta V}\right) \\
& \times \int\left(\frac{N_{\mathrm{HI}}(\boldsymbol{\beta})}{10^{22} \mathrm{~cm}^{-2}}\right) \mu(\boldsymbol{\beta}) d^{2} \beta .
\end{aligned}
$$

This can be expressed in terms of the flux of the unlensed source

$$
F_{\nu}[\text { Lensed }]=F_{\nu}[\text { Source }] \times \bar{\mu}
$$


where $\bar{\mu}$ is the magnification averaged over the angular extent of the source

$$
\bar{\mu}(\boldsymbol{\beta}, R)=\int d^{2} \beta^{\prime} \mu\left(\boldsymbol{\beta}-\boldsymbol{\beta}^{\prime}\right) W\left(\boldsymbol{\beta}^{\prime}, R\right) .
$$

The normalized window function $W(\boldsymbol{\beta}, R)$ represents the radial profile of the HI cloud and for the UD and ED models we use

$$
\begin{aligned}
W\left(\boldsymbol{\beta}, r_{\mathrm{DLA}}\right) & =\frac{d_{s}^{2}}{\pi r_{\mathrm{DLA}}^{2}} \Theta\left(|\boldsymbol{\beta}|-\frac{r_{\mathrm{DLA}}}{d_{s}}\right) \\
W\left(\boldsymbol{\beta}, R_{d}\right) & =\frac{d_{s}^{2}}{2 \pi R_{\mathrm{d}}^{2}} \exp \left(-\frac{|\boldsymbol{\beta}|}{R_{d} / d_{s}}\right) \quad(\mathrm{ED}),
\end{aligned}
$$

where $\Theta$ is the Heaviside step function. In our calculations we have evaluated $\bar{\mu}(\boldsymbol{\beta}, R)$ by first calculating $\mu(\boldsymbol{\beta})$ on a finely-spaced grid in the source plane. This is then convolved with the window function using the Fast Fourier Transform algorithm (Press et al. 1992) to obtain $\bar{\mu}(\boldsymbol{\beta}, R)$. We have tested the convergence of this procedure with respect to variation of the grid size. In situations where there are multiple images we have added up the magnification of all the magnified images; because the typical image separation is less than the beam width of the GMRT and the observations which we are discussing here will not be able to resolve the multiple images.

We use the procedure discussed above to calculate $\bar{\mu}(\boldsymbol{\beta}, R)$ on a grid. This is used to calculate $A\left(>\bar{\mu}_{0}\right)\left(\bar{\mu}_{0}\right.$ is any fiducial average magnification) which is the area (in arc-second ${ }^{2}$ ) in the source plane for which the condition $\bar{\mu}(\boldsymbol{\beta}, R)>\bar{\mu}_{0}$ is satisfied. For observations with a detection threshold of $F_{\nu}$ [min], a HI cloud with unlensed flux $F_{\nu}$ will have a lensed flux greater than $F_{\nu}$ [min] for any position within the area $A\left(>F_{\nu}[\mathrm{min}] / F_{\nu}\right)$. Summing up the contributions from HI clouds with all possible values of the unlensed flux gives us $\mathcal{N}\left(>F_{\nu}[\mathrm{min}]\right)$ the total number of HI clouds that can be detected in the field of a cluster

$$
\mathcal{N}\left(>F_{\nu}[\min ]\right)=\int d F_{\nu} \frac{d^{2} N}{d \Omega d F_{\nu}} A\left(>F_{\nu}[\min ] / F_{\nu}\right)
$$

It should be pointed out that throughout the analysis we have assumed all the HI clouds to be oriented face-on. In reality the HI clouds will occur at all possible angles. In this case the mean magnification $\bar{\mu}(\boldsymbol{\beta}, R)$ will differ with the orientation of the cloud. However, the projected

area is maximum when the cloud is face-on and the mean magnification will be lower relative to the situation where the cloud is located at the same position with an edge-on orientation. This means that our analysis gives an underestimate of the magnification and we may expect larger magnifications when all possible orientations are taken into account.

\section{Results}

We first discuss our results for observations centered at $320 \mathrm{MHz}$. The results for 620 and $233 \mathrm{MHz}$ are summarized at the end of this section. 
We have calculated $A(>\bar{\mu})$ and $\mathcal{N}\left(>F_{\nu}[\min ]\right)$ for several cluster parameters with the disk parameters varying over the allowed range discussed earlier for the UD and ED models. The effect of varying the cluster parameters is to change $A(>\bar{\mu})$ which in turn affects the number of detected HI clouds. The regions of high magnification $(\mu>10)$ are restricted along the caustics and all the contribution to $A(>\bar{\mu})$ is from a narrow strip along the caustics. Although the length of the caustic curve increases as the ellipticity $\epsilon$ is increased, this is accompanied by a fall in the values of the magnification. The value $\epsilon=0.1$ is a good compromise between these two effects and the results are presented for this value. The $\epsilon$ dependence of the results is rather weak in the range $0.1 \leq \epsilon \leq 0.3$. The values of $A(>\bar{\mu})$ and $\mathcal{N}\left(>F_{\nu}[\mathrm{min}]\right)$ decrease by around $10 \%$ as the value of $\epsilon$ changes from 0.1 to 0.3 . Amongst the cluster parameters, changing the velocity dispersion $\sigma$ has the strongest effect on $A(>\bar{\mu})$ and this is shown in Figures 2 and 3. Varying the cluster redshift from 0.1 to 0.5 causes $A(>\bar{\mu})$ to decrease by nearly $30 \%$.

Varying the radius of the HI clouds has a few distinct effects. First, an increase in the size of the HI clouds decreases the average magnification $\bar{\mu}(\boldsymbol{\beta}, R)$. The area $A(>\bar{\mu})$ also decreases as the radius of the HI cloud is increased and this is clearly seen in Figures 2 and 3. The second effect is that a larger cloud radius gives a larger value of the unlensed flux (Eqs. (3) and (8)). The third effect is that as the radius of the HI clouds is increased, the number of clouds in the field decreases. This is seen in Figure 1 for the UD model. All these effects combine to determine how the number of detected HI clouds depends on the radius of the clouds. As is seen in Figure 4 for the UD model, an increase in the cloud radius generally increases the number of detected HI clouds. Only for large values of $r_{\text {DLA }}$ do we find a decrease in the number of HI clouds causing a decrement in the number of detections. The results for the ED model shown in Figure (5) are qualitatively similar to those for the UD model. In this case the parameter that controls the number of clouds in a field is $N_{\mathrm{HI}}(0)$, and the number of clouds in a field falls as $N_{\mathrm{HI}}(0)$ is increased (Figure 1). As in the UD case, this generally leads to an increase in the number of detected clouds because a fall in the number of clouds is often over-compensated by an increase in the unlensed flux. An important parameter in both the cloud models is the spectral index, $\alpha$ (Eqs. (10) and (11)). The number of detected clouds decreases by $\lesssim 25 \%$ as $\alpha$ is increased from 0.5 to 1 .

For a detection threshold of $50 \mu \mathrm{Jy}$, for observations centered on a cluster with $\sigma=1200 \mathrm{~km} \mathrm{~s}^{-1}$, the expected number of detected clouds is in the range 0.1 to 0.5 for $r_{\text {DLA }} \geq 10 \mathrm{kpc}$ in the UD model and for $N_{\mathrm{HI}}(0) \geq 5 \times 10^{21} \mathrm{~cm}^{-2}$ in the ED model. For both the UD and the ED models the lower limit for detection approximately corresponds to the mass range $5 \times 10^{8} \mathrm{M}_{\odot} \leq M_{\mathrm{HI}} \leq 2.5 \times 10^{10} \mathrm{M}_{\odot}$.

The number of detections will go up if the observations are centered on a cluster with a higher velocity dispersion, since $\mathcal{N}(>50 \mu \mathrm{Jy})$ goes up by a factor of 2 to 3 if $\sigma=1800 \mathrm{~km} \mathrm{~s}^{-1}$ instead of $\sigma=1200 \mathrm{~km} \mathrm{~s}^{-1}$ (Figures 4 and 5). In addition it will be possible to detect the HI clouds even if they have lower HI masses.

Future radio telescopes will reach sensitivities of around $2 \mu \mathrm{Jy}$. Eqs. (3) and (8) show that it may then be possible to detect the HI clouds without the aid of gravitational lensing. In the UD 
model if $r_{\text {DLA }} \geq 20 \mathrm{kpc}$ then a substantial fraction of the HI clouds will be detected without any gravitational lensing. While a small fraction of the HI clouds may be detected without gravitational lensing if $10 \mathrm{kpc}<r_{\text {DLA }}<20 \mathrm{kpc}$, it will not be possible to observe the clouds without the aid of gravitational lensing if $r_{\text {DLA }} \leq 10 \mathrm{kpc}$. Figure 6 shows that observations with a detection threshold of $2 \mu \mathrm{Jy}$ centered on a cluster with $\sigma=1200 \mathrm{~km} \mathrm{~s}^{-1}$ are expected to have a few detections even if the cloud radius is as small as $r_{\text {DLA }}=2 \mathrm{kpc}$. This corresponds to a mass range $2 \times 10^{7} \mathrm{M}_{\odot} \leq$ $M_{\mathrm{HI}} \leq 10^{9} \mathrm{M}_{\odot}$.

The discussion until now has been restricted to $320 \mathrm{MHz}$. The main differences which arise at other frequencies are: (a) the flux from HI clouds changes (Eq. (2)), (b) the total number of clouds is different owing to changes in $\Omega_{\mathrm{HI}}$ (Eq. (6)) and $\Delta z$ and, (c) the magnification of sources changes owing to change in the source redshift $(\S 3)$.

At $z \simeq 1.3(610 \mathrm{MHz})$ observations indicate $\alpha \simeq 2.5$ and $N_{\mathrm{HI}}[\max ] \sim 10^{21} \mathrm{~cm}^{-2}$ (Lanzetta et al. 1995). Also, $\Omega_{\mathrm{HI}}$ falls considerably from its value at $z \simeq 3.4$ However, owing to obscuration of quasar light by the dust present in HI clouds (Fall \& Pei 1995) these values may not be representative of the population of HI clouds and it is possible that the observed $\Omega_{\mathrm{HI}}$ is underestimated by a factor of 3 (Fall \& Pei 1995) at this redshift. Given the uncertainty in the HI content we have used several values of spectral indices and $\Omega_{\mathrm{HI}}$. Figure (7) shows the results for just one set of cluster parameters.

The value of $\Omega_{\mathrm{HI}}$ at $z \simeq 5.10(233 \mathrm{MHz})$ is not known. For the purposes of this paper we use the value given by Storrie-Lombardi et al. (1996) which is valid for $z \leq 4.7$. For $\nu=233 \mathrm{MHz}$ the number of detectable clouds in a cluster field is shown in Figure (7).

\section{Discussion and Conclusions}

In this paper we have investigated the possibility of detecting the redshifted $21 \mathrm{~cm}$ emission from HI clouds at high redshift. Such observations are not possible with existing radio telescopes unless we observe the cloud through a cluster gravitational lens which magnifies the HI flux. However, this method has the disadvantage that it will work only for clouds which lie very close to the caustics of cluster lenses.

Given the lack of a clear picture about the nature of these objects we have used two simple models, namely the UD and the ED models $(\S 2)$. The results are similar for both the models (Figures 4 and 5). Both the models have free parameters which effectively allow us the freedom of distributing the total $\mathrm{HI}$ available in the $\mathrm{HI}$ clouds at high redshift into either a large number of clouds with a small amount of HI each or a small number of clouds with large amounts of HI each. In the former scenario the probability of a cloud being located very close to the caustic of a gravitational lens and experiencing a large magnification is higher but this is offset by the fact that the unlensed flux of the HI clouds will be very small and a magnification of $\simeq 50$ may not be sufficient to render the lensed flux above the threshold value for detection. The parameter range 
where the probability of a detection is maximum changes with the threshold flux and this may lead to the possibility of using such observations to constrain the distribution of the HI masses of the clouds. This possibility has not been studied here in any detail.

We find that for observations at $320 \mathrm{MHz}$ with a detection threshold of $50 \mu \mathrm{Jy}$ centered on a cluster with $\sigma=1200 \mathrm{~km} \mathrm{~s}^{-1}$, the chance of detecting a HI cloud is greater than $10 \%$ provided the minimum $\mathrm{HI}$ content of these clouds is in the mass range $5 \times 10^{8} \mathrm{M}_{\odot} \leq M_{\mathrm{HI}} \leq 2.5 \times 10^{10} \mathrm{M}_{\odot}$. The chances of detecting an HI cloud increases for a cluster with a higher velocity dispersion, and a single deep image in the field of a cluster such as Abell 1689 (Tyson et al.1990; Pierre et al.1991; Smail et al.1991), which has $\sigma=1989 \mathrm{~km} \mathrm{~s}^{-1}$ and $z_{l}=0.196$, might either reveal a cloud or put meaningful bounds on the mass range and mass spectral index $\alpha$ of the HI clouds.

At $320 \mathrm{MHz}$ the GMRT will reach a sensitivity of $50 \mu \mathrm{Jy}$ with $100 \mathrm{hrs}$ of integration at a frequency resolution of $\Delta \nu=1.25 \times 10^{5} \mathrm{~Hz}$ corresponding to a velocity width $\Delta V \simeq 115 \mathrm{~km} \mathrm{~s}^{-1}$. An alternative strategy would be to observe several clusters to a threshold flux of $100 \mu \mathrm{Jy}$. The latter strategy may be superior for a part of the parameter range shown in Figures 4, 5 and 7, i.e. the number of expected detections will be higher for the same total observation time.

Future radio telescope Square Kilometer Array $(\mathrm{SKA})$ will reach sensitivity $\simeq 2 \mu \mathrm{Jy}$ at $\nu \simeq$ $320 \mathrm{MHz}$ for $\Delta V \simeq 200 \mathrm{~km} \mathrm{~s}^{-1}$ in an integration time of $\simeq 8 \mathrm{hrs}^{1}$. In this case observations centered on clusters with $\sigma=1200 \mathrm{~km} \mathrm{~s}^{-1}$ are expected to detect a few HI clouds even if the clouds have low $\mathrm{HI}$ masses in the range $2 \times 10^{7} \mathrm{M}_{\odot} \leq M_{\mathrm{HI}} \leq 10^{9} \mathrm{M}_{\odot}$.

Our results indicate that the probability of detecting a gravitationally lensed HI cloud at 233 and $610 \mathrm{MHz}$ is generally lower than the probability at $320 \mathrm{MHz}$. However, it should be borne in mind that there are larger uncertainties in our predictions at 233 and $610 \mathrm{MHz}$ as compared to the predictions for $320 \mathrm{MHz}$.

We conclude by noting that the strategy of carrying out deep radio observations centered on known cluster lenses will make it possible to detect the redshifted $21 \mathrm{~cm}$ emission from lensed HI clouds with either the GMRT or the SKA, depending on the HI content of these clouds. Such observations will shed new light on these objects whose HI content has been observed only in absorption to date.

The authors would like to thank Jasjeet Bagla, Jayaram Chengalur, Divya Oberoi, and Somak Raychaudhury for useful discussions and an anonymous referee for helpful comments. T.D.S thanks CSIR for providing financial support.

\footnotetext{
${ }^{1}$ for details see http://www.nfra.nl/skai/science
} 


\section{Appendix A}

Here we present a derivation of Eq. (1) which gives the specific intensity of the redshifted HI emission from a cloud which has $\mathrm{HI}$ column density $N_{\mathrm{HI}}$, velocity dispersion $\Delta V$ and is at a redshift $z$.

The HI contained in a part of the cloud which subtends a solid angle $\Delta \Omega$ at the observer is $N_{\mathrm{HI}} r_{A}^{2}(z) \Delta \Omega$, where $r_{A}^{2}(z)$ is the angular diameter distance to the HI cloud. In the rest frame of the cloud the total luminosity of the HI emission is

$$
\Delta L=A_{21} h_{P} \nu_{e} f N_{\mathrm{HI}} r_{A}^{2}(z) \Delta \Omega
$$

and it will be distributed over the frequency interval $\Delta \nu_{e}=\Delta V \nu_{e} / c$. Here $\nu_{e}=1420 \mathrm{MHz}$ is the rest frame frequency of the $\mathrm{HI}$ emission, $h_{P}$ is the Planck constant, $f$ is the fraction of $\mathrm{HI}$ atoms in the excited state and $A_{21}=2.85 \times 10^{-15} s^{-1}$ is the Einstein coefficient for the spontaneous emission.

At the observer the radiation will be redshifted to a frequency $\nu=\nu_{e} /(1+z)$ and it will be distributed over the frequency interval $\Delta \nu=\Delta \nu_{e} /(1+z)$ The flux $\Delta F$ is given by

$$
\Delta F=\frac{\Delta L}{4 \pi r_{L}^{2}(z)}
$$

where $r_{L}(z)$ is the luminosity distance to the HI cloud. We use this to calculate the specific intensity

$$
I_{\nu}=\frac{\Delta F}{\Delta \nu \Delta \Omega}
$$

Using Eqs. (18) and (19) and the relation (see e.g. Peebles 1993)

$$
\frac{r_{A}(z)}{r_{L}(z)}=(1+z)^{-2}
$$

we obtain

$$
I_{\nu}=\frac{N_{\mathrm{HI}} f A_{21} h_{P} c}{4 \pi(1+z)^{3} \Delta V}
$$

In a situation where the spin temperature $T_{s}$ is high $\left(T_{s} \gg h_{P} \nu_{e} / k_{B}\right)$ we have $f=3 / 4$, where $k_{B}$ denotes the Boltzmann constant. Equation (22) can be evaluated to give

$$
I_{\nu}=40 \mu \mathrm{Jy} \operatorname{arcsec}^{-2}(1+z)^{-3}\left(\frac{N_{\mathrm{HI}}}{10^{22} \mathrm{~cm}^{-2}}\right)\left(\frac{200 \mathrm{~km} \mathrm{~s}^{-1}}{\Delta V}\right) .
$$

\section{REFERENCES}

Briggs, F. H., Wolfe, A. M., Liszt, H.S., Davis, M. M., \& Turner, K. L. 1989, ApJ, 314, 650 
Briggs, F. H. 1988, QSO absorption lines: Probing the universe, p. 275-290, Cambridge University Press

Djorgovski, S. G., Pahre, M. A., Bechtold, \& J., Elston, R. 1996, Nature, 382, 234

Fall \& Pei 1995, QSO Absorption Lines, ed. G. Meylan, Springer-Verlag

Fynbo, J. U., Burud, I., \& Moller, P. 2000, A \& A, 358, 48

Fynbo, J. U., Moller, P, \& S. J. Warren 1999, MNRAS, 305, 849

Haehnelt, M., Steinmetz, M., \& Rauch, M. 1998, ApJ, 495, 647

Kassiola, A. \& Kovner, I. 1993, ApJ, 417, 450

Kulkarni, V. P. et al. 2000, ApJ, 536, 36

Lane, W. M., Briggs, F. H. \& Smette, A. 2000, ApJ, 532, 146

Lanzetta, K. M., Wolfe, A. M., \& Turnshek, D. A. 1995, ApJ, 430, 435

Le Brun, V, Bergeron, J. \& Boissé, 1997, A \& A, 321, 733

Mazure, A. et al. 1996, A \& A, 310, 31

Moller, P. \& Warren, S. J. \& Fynbo, J. U. 1998, A \& A, 330, 19

Moller, P. \& Warren, S. J. 1993, A \& A, 270, 43

Peebles, P. J. E. 1993, Principles of Physical Cosmology, Princeton university Press

Pierre, M., Soucail, G., Mellier, Y. \& Sanahuja, B., 1991, ApJ, 366, 405

Press, W. H., Teukolsky, S., Vellering, W. T., \& Flannery, B.P. 1992, Numerical Recipes in Fortran, Cambridge university Press

Prochaska, J. X. \& Wolfe, A. M. 1998, ApJ, 507, 113

Schneider, P., Ehlers, J., Falco, E. E., 1992, Gravitational Lenses, Springer-Verlag

Smail, I., Ellis, R. S., Fitchett, M. J., Norgaard-Nielsen, H. U., Hansen, L., Jorgensen, H. E., 1991, MNRAS, 252, 19

Smette, A., 1995, QSO Absorption Lines, ed. G. Meylan, Springer-Verlag

Storrie-Lombardi, L.J., McMahon, R.G., Irwin, M.J. 1996, MNRAS, 283, L79

Swarup, G., Ananthakrishan, S., Kapahi, V. K., Rao, A. P., Subrahmanya, C. R., \& Kulkarni, V. K. 1991, Curr. Sci., 60, 95 
Tyson, J. A., Valdes,F. and Wenk, R.A., 1990, ApJ. Lett., 349, 1.

Warren, S. J. \& Moller, P. 1996, A \& A, 311, 25

Wolfe, A. 1995, QSO Absorption Lines, ed. G. Meylan, Springer-Verlag 


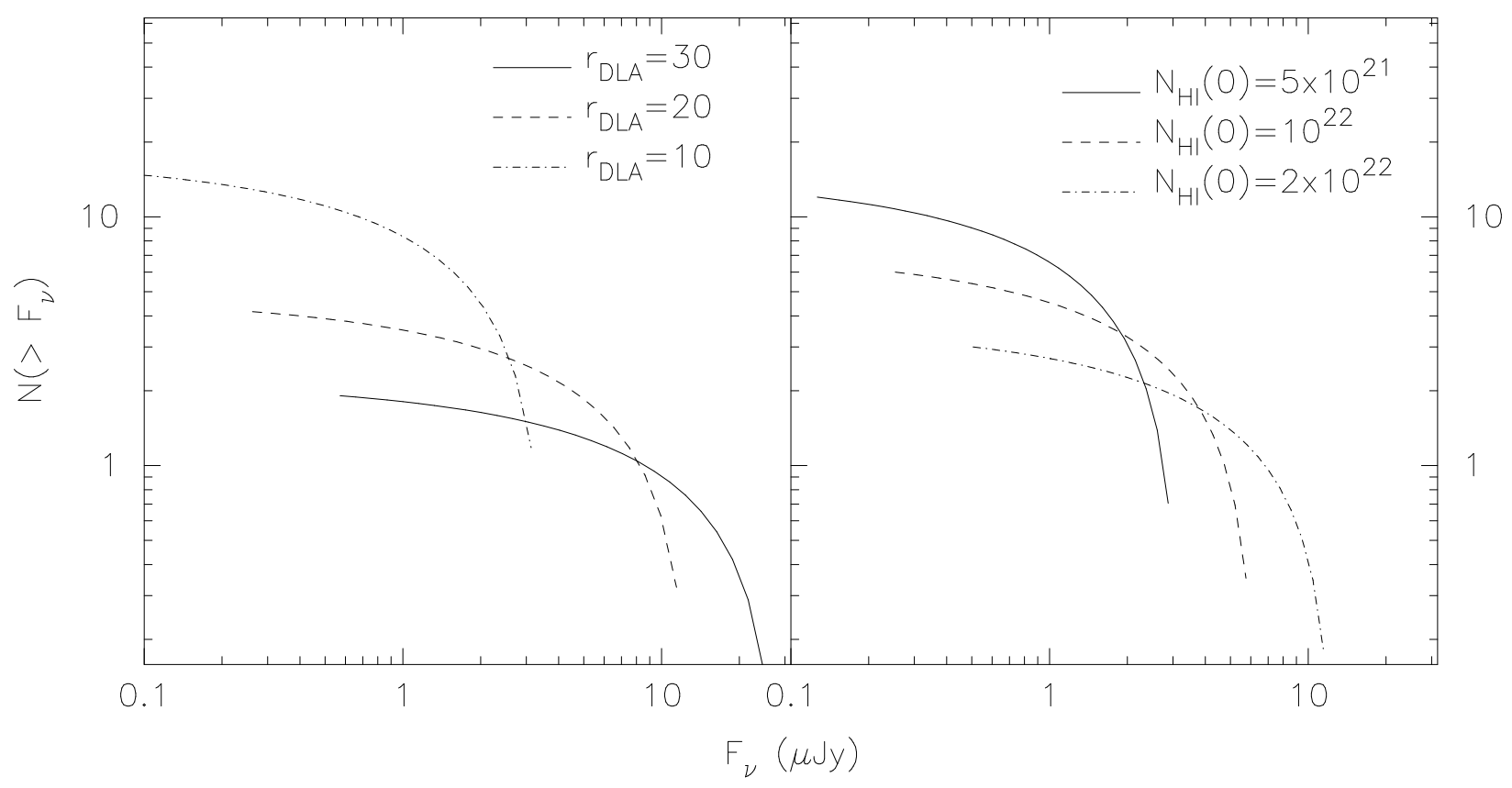

Fig. 1. - This shows the number of HI clouds with flux greater than $F_{\nu}$ in a $1^{\prime} \times 1^{\prime}$ patch of the sky for a bandwidth of $16 \mathrm{MHz}$ centred at $320 \mathrm{MHz}$, and a mass spectral index $\alpha=0.5$. Left Panel is the UD model for three values of $r_{\text {DLA }}$ (in kpc). Right Panel is the ED model for three values of $N_{\mathrm{HI}}(0)$. 


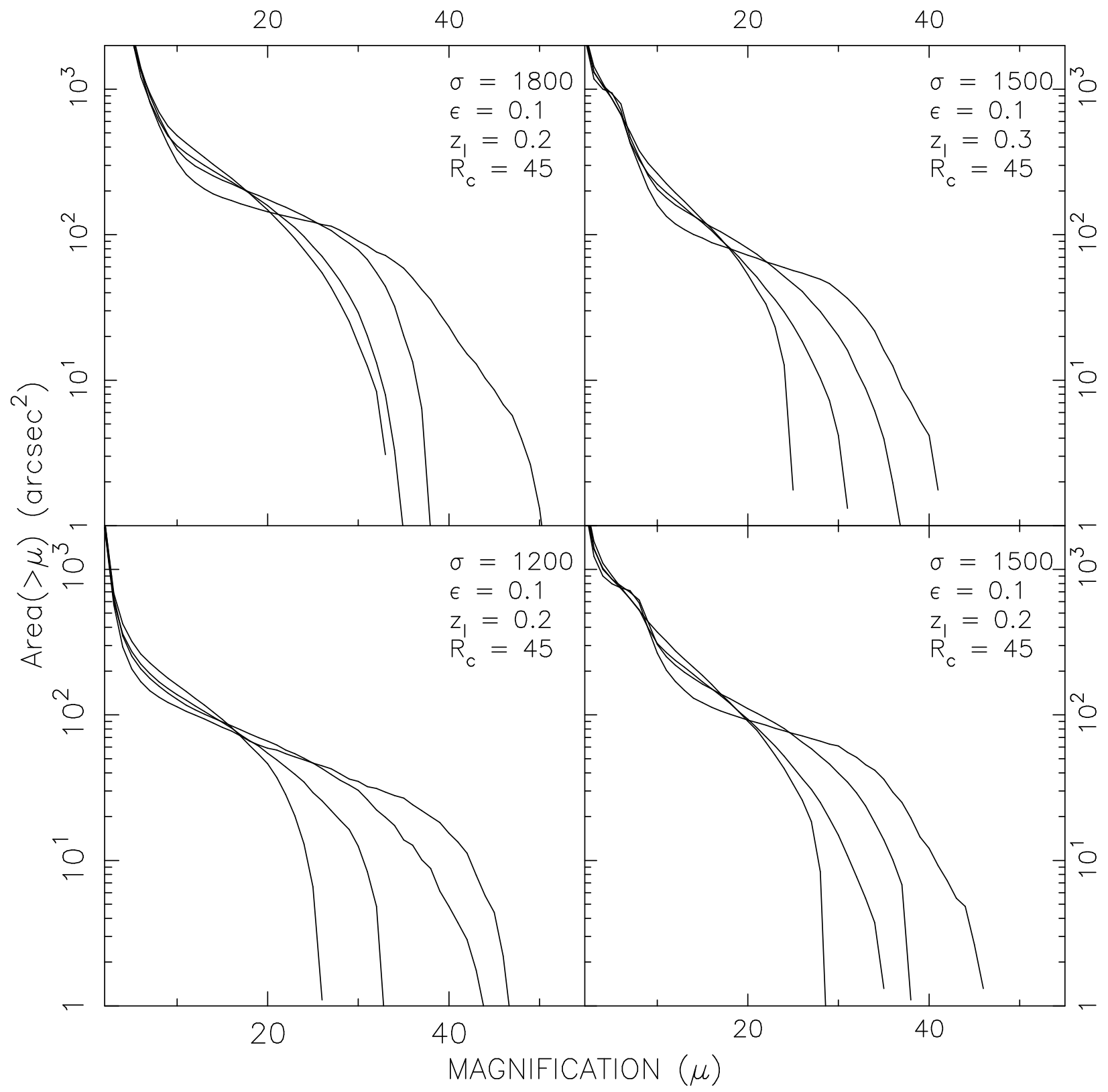

Fig. 2.- The area in which the averaged amplification $\bar{\mu}$ exceeds a given value $\mu$ is shown for several cluster parameters and clouds radii in the the UD model. In each panel, the clouds radius $r_{\text {DLA }}=\{10,20,30,40\} \mathrm{kpc}$ corresponds to curves of decreasing maximum amplification. 


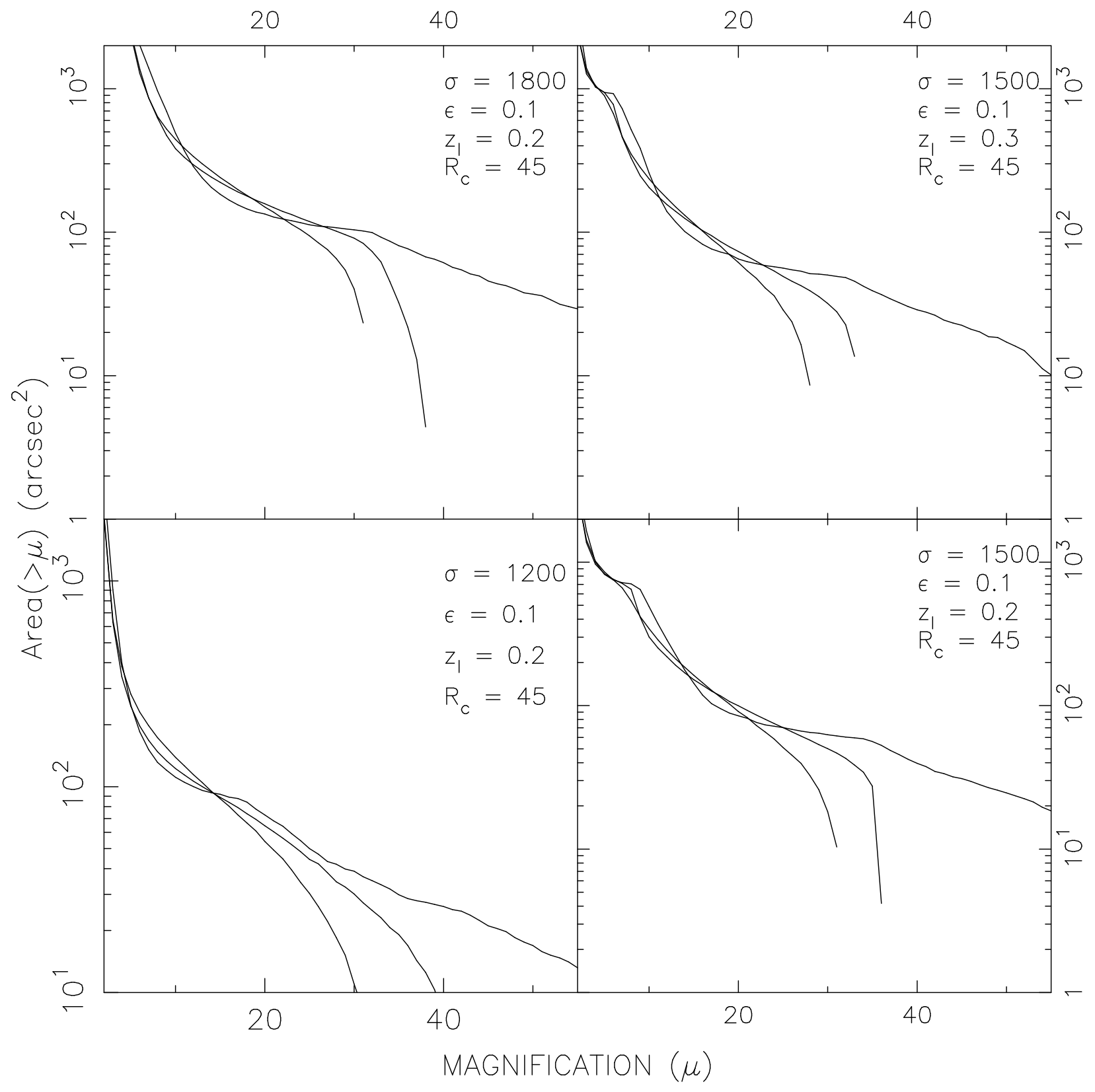

Fig. 3. - The area in which the averaged amplification $\bar{\mu}$ exceeds a given value $\mu$ is shown for several cluster parameters and radius parameter $R_{d}$ in the the ED model. In each panel, the radius parameter $R_{d}=\{2,8,14\} \mathrm{kpc}$, corresponding to curves of decreasing maximum amplification. 


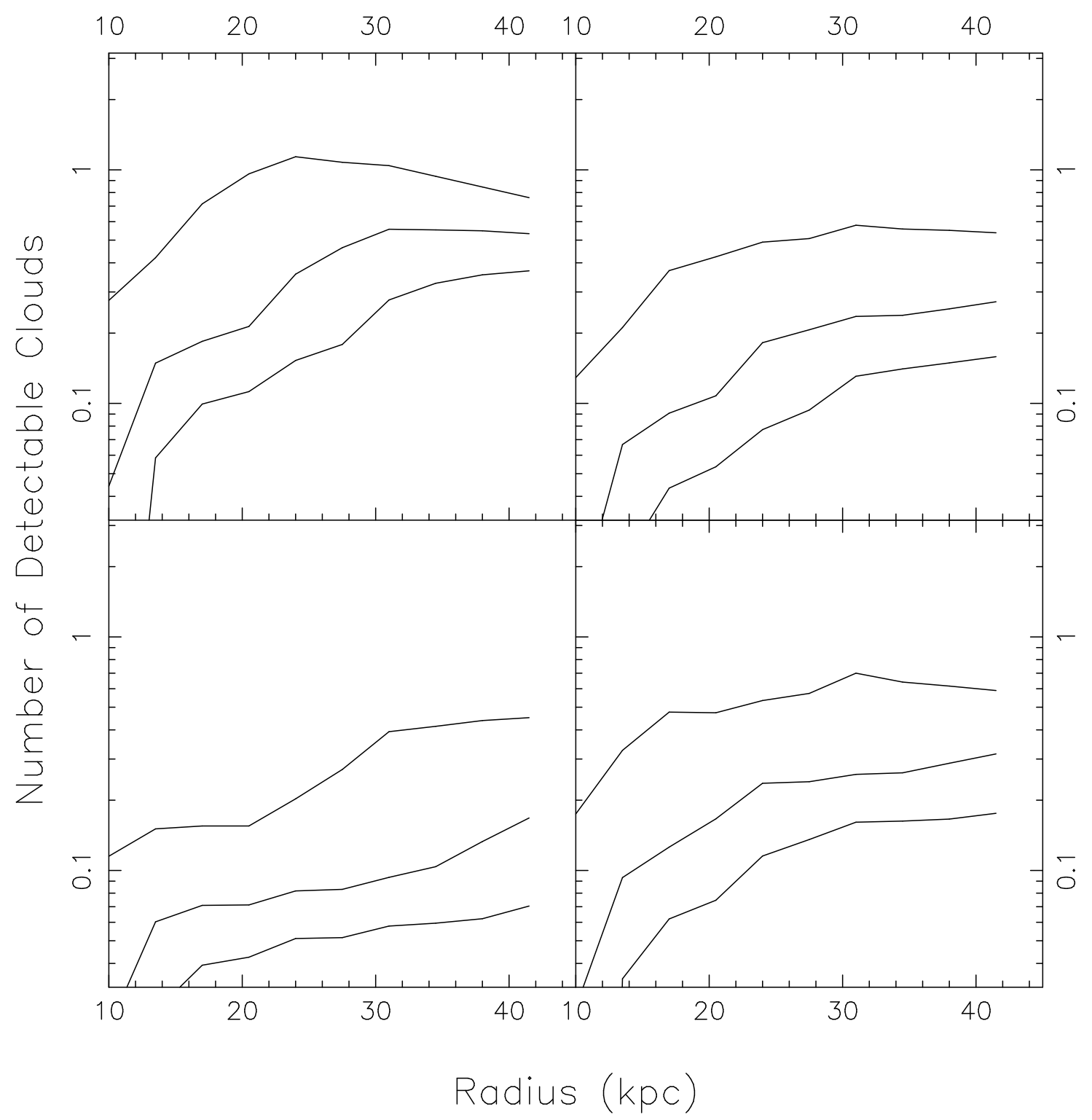

Fig. 4.- This shows the number of lensed HI clouds expected to be detected in the field of a cluster as a function of the cloud radius in the UD model with $\alpha=0.5$, for the 4 cluster models shown in Figure 2. The three curves in each panel correspond to detection thresholds $F_{\nu}[\mathrm{min}]=\{50,100,150\} \mu \mathrm{Jy}$, with the number of expected detections decreasing for higher flux thresholds. 


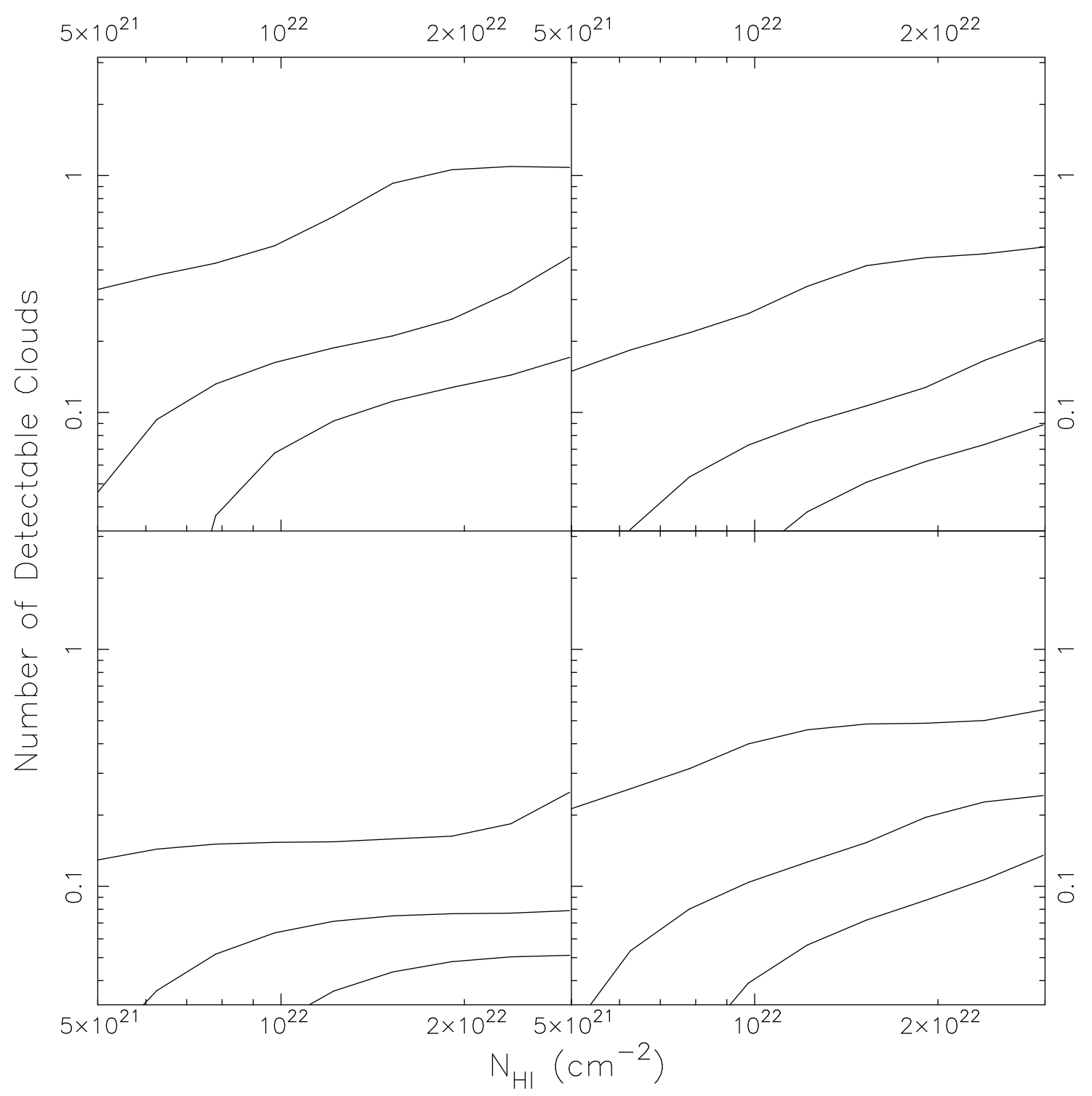

Fig. 5.- This shows the number of lensed HI clouds expected to be detected in the field of a cluster as a function of the central column density $N_{\mathrm{HI}}(0)$ in the ED model with $\alpha=0.5$. for the 4 cluster models shown in Figure 3. The three curves in each panel correspond to detection thresholds $F_{\nu}[\mathrm{min}]=\{50,100,150\} \mu \mathrm{Jy}$ with the number of expected detections decreasing for higher flux thresholds. 


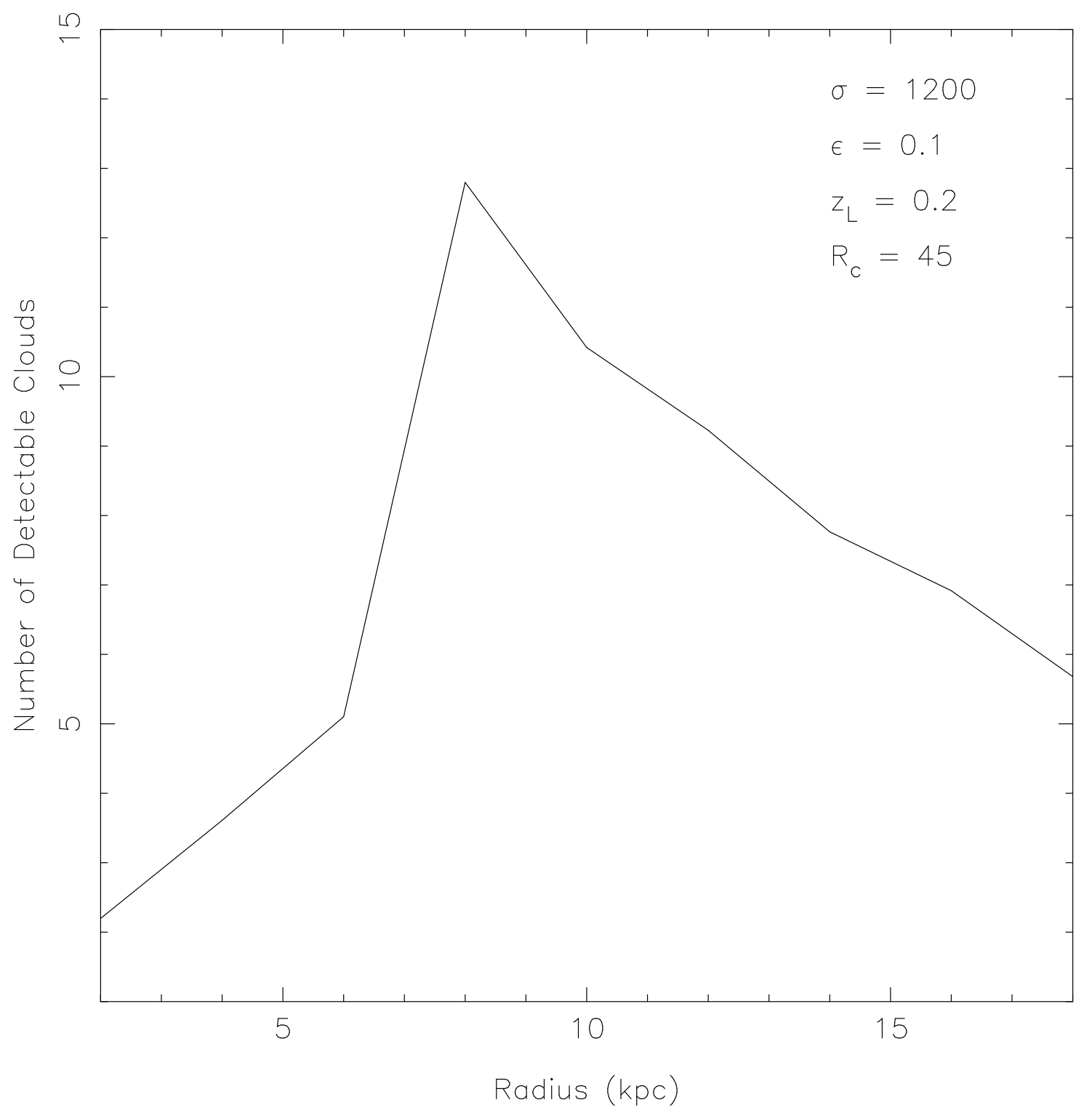

Fig. 6. - This shows the number of lensed HI clouds expected to be detected in the field of a cluster as a function of the cloud radius, $r_{\mathrm{DLA}}$, in the UD model with $\alpha=0.5$ for observations with $F_{\nu}[\mathrm{min}]=2 \mu \mathrm{Jy}$ and a bandwidth of $16 \mathrm{MHz}$ centred at $320 \mathrm{MHz}$. The cluster parameters are shown in the figure. 


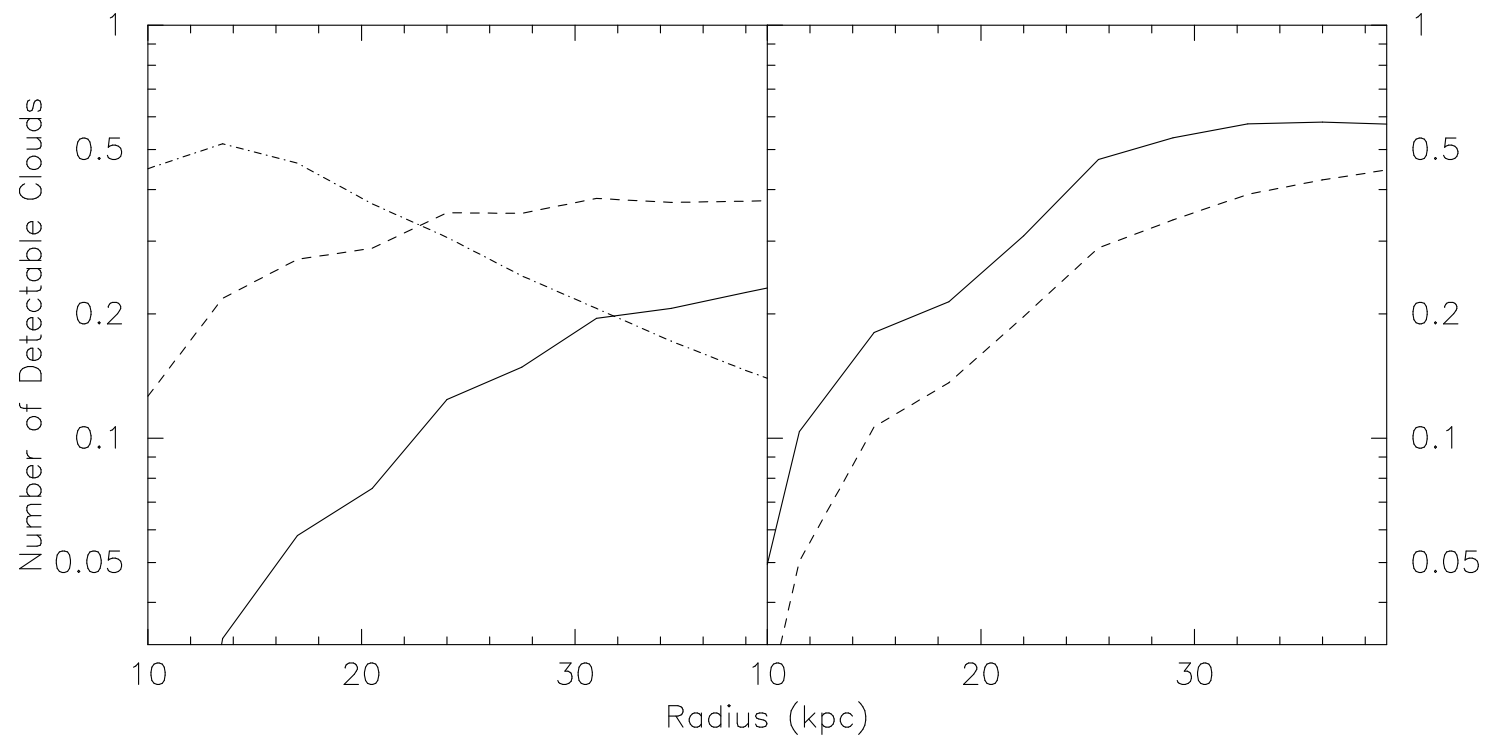

Fig. 7.- This shows the number of lensed HI clouds expected to be detected in the field of a cluster with $\sigma=1800 \mathrm{~km} \mathrm{~s}^{-1}, \epsilon=0.1, z_{l}=0.2$ and $R_{c}=45 \mathrm{kpc}$ in the UD model. Left Panel is at $610 \mathrm{MHz}$. The solid curve is for $N_{\mathrm{HI}}[\max ]=10^{21} \mathrm{~cm}^{-2}, \alpha=2.3$ and $\Omega_{\mathrm{HI}}$ given by Eq. (6). The dashed (dot-dashed) curve uses $\alpha=2.3(\alpha=0.5)$ with $\Omega_{\mathrm{HI}}$ twice the prediction of eq. (6) and $N_{\mathrm{HI}}[\max ]=10^{22} \mathrm{~cm}^{-2}$ Right Panel is at $233 \mathrm{MHz}$ with $\alpha=0.5$ (solid line) and $\alpha=1.5$ (dashed line) 